\title{
Failure of Humanities-based Instruction to Achieve Students' Language Goal in College English Courses
}

\author{
Fuhua Liu \\ School of Foreign Languages, Dalian Jiaotong University, Dalian, Liaoning 116028, China
}

Keywords: failure, humanities-based instruction, language goal, content-based instruction, theme-based instruction

Abstract: The present paper is a detailed analysis of failure of humanities-based instruction to achieve students' language goal in college English courses. The essence of contentbased instruction and the phenomenon of applying humanities-based instruction to achieve students’ language goal are briefly introduced; the reasons for the failure are analyzed; corresponding remedies are offered.

\section{Introduction}

Content-based instruction or CBI for short refers to the shift of focus from language-based learning and teaching to content-based learning and teaching that naturally induce language acquisition [1-3]. Therefore, CBI is about language acquisition rather than content or knowledge acquisition. Just as Chen Dongchun claims, "The core of CBI is about language learning and teaching" [4]. In appearance, CBI indicates the change of teaching methods and teaching modes. But in essence, it is a learning and teaching concept as to how to learn a foreign language efficiently and effectively.

Many foreign language universities or departments have turned to content-based instruction or CBI to enhance students' language abilities. Some college English teachers even establish teams and apply for province-level and state-level research projects to explore this teaching method. While most universities or departments use theme-based instruction, a sub-type of CBI, in a bid to improve students' English proficiency, especially their output-oriented proficiency in real communicative situations, some universities or departments take a step further, intending to improve students' language abilities through the study of humanistic subjects such as history, geography and religion of some major foreign countries or Chinese culture. Despite the occurrence of application of humanities-based instruction in reality, I firmly hold the belief that the systematic study of humanities is never a proper means for college students to achieve their language goal. 


\section{Body}

\subsection{The Infeasibility of Applying Humanities-based Instruction to Achieve Students'}

\section{Language Goal}

Then come to the critical question. Is it feasible to apply humanities-based instruction to achieve students' language goal? Personally, I don't think humanities will work well for the following reasons. First, students can hardly be inspired to use related language as in reality the chances of coming into contact with a certain humanistic subject are slim. Relatively speaking, it is kind of insignificant for college students to pour too much time and energy into the study of foreign humanities such as American history or Australian geography. There is no denying the fact that the more knowledge college students gain, the more comprehensive their abilities will become. With a wide range of knowledge in mind, students will enrich their inner world and enjoy more opportunities to establish ties with foreign friends. But in reality, the chances for students to talk about humanities are slim. Even with the advancement of some online communication platforms on the Internet, students still have difficulty finding ideal partners to talk about humanistic knowledge they have acquired. The slim chances of talking about humanities in English in reality discourage students to put efforts in class. Without enough language practice on the required humanistic topic, their language abilities fade away. As a result, the students taking the humanistic approach may end up benefiting little linguistically. They might have learned some knowledge about a humanist subject, and they might also be capable of doing objective questions in a test concerning the subject, but they might have trouble expressing the ideas about the specialized field for the lack of two-way communication opportunities.

Second, as part of social sciences, humanistic subjects are specialized and systematic studies. Some contents are so complicated that learners may have trouble understanding them even in their native tongue. The biggest barrier is specialized vocabulary. Unlike theme-based instruction in which weird words can always be replaced by other common words or expressed in several other ways to make the speaker understood, humanities-based instruction has many specialized terms that speakers sometimes cannot find proper substitutes. To memorize a large number of specialized terms in a certain humanist subject, students have to spend a lot of time on the memorizing activities but end up finding that they just cannot pronounce and spell these terms accurately. What's worse, the specialized terms have actually prevented students from practicing commonlyused vocabulary and sentence structure. All in all, instead of facilitating the development of students' language abilities, humanities-based instruction actually hinders students from achieving their language goal. The possible result is that it is probably easy for the majority of college students to take in what they have learned about a humanistic subject, but they may find it extremely difficult to express out the whole meaning on a certain humanistic topic in English.

I conducted a small-sized survey by talking about humanities-based instruction with 13 of my students in private. The result of the survey goes as follows. 12 out of the 13 students believe it is practically impossible to greatly improve their English by taking humanities-based courses such as Australian geography or Chinese culture. 2 students mention that even their teachers have trouble pronouncing accurately some specialized words and expressing precisely some complex ideas without reference to textbooks. 10 of them predict that they will forget most of what they have learned in a year. However, all 13 do admit that such courses have broadened their horizon and gained knowledge about a certain field. Therefore, in practice it also proves that humanities-based instruction does not work in improving students' language abilities. If some schools or departments do want to use humanities-based instruction to improve students' English abilities, the possible result will be more of the improvement of thought or knowledge than language itself. 
Since humanities-based instruction cannot efficiently and effectively enhance students' language abilities, why do some schools or departments still pursue this sub-type of CBI? Of course, some schools or departments make use of humanities-based instruction of CBI for the benefit of teachers themselves. It is teachers who benefit most from the exploitation of such teaching methods. They tend to use CBI theory to apply for some research projects and publish papers.

Actually, the content-based learning and teaching of the advanced stage is a natural development from language learning and teaching of the preliminary stage. The two stages of learning and teaching have different focuses, with the former focused on "content" and the latter on "language". The language goal in the preliminary stage can never be achieved efficiently and effectively through the use of the method in the advanced stage. In a word, humanities-based instruction goes against the original aspiration of CBI's language goal and essence.

\subsection{The Remedies for the Misunderstandings and Misuse of Humanities-based Instruction}

What are the remedies for the misunderstandings and misuse of humanities-based instruction? On the one hand, for the language goal in the preliminary stage, theme-based instruction is always a super choice for college students who have already had solid language foundation. Wang Shixian believes the theme mode of CBI favors the language goal [5]. Theme-based instruction tends to use relatively simple, general-knowledge-oriented and interesting materials for students' language study. The content of theme-based instruction fits students' language goal in that it well enables them to enhance their language input such as listening, viewing and reading and output such as speaking, writing and translating for its relative simplicity. Students also manage to conduct various activities such as discussion, debate and role-play by employing the theme mode of CBI. Equally important is that the abundance of raw materials from various fields of science and humanities ensures students the wide range of knowledge to be taken.

For the content goal in the advanced stage, it is highly suggested that students choose one particular discipline and keep focusing on it for preparation of their future career or for pure interest. As students' energy and time are limited, they cannot excel in all humanistic subjects. They are advised to have a general knowledge of all subjects, but choose the one they like and focus on it. They should end up having a general understanding of all humanistic subjects with the help of dictionaries and at the same time being able to communicate in written form or orally about a specialized subject that they have chosen.

\section{Conclusion}

The present paper first clarifies the definition of CBI, a learning and teaching method aiming to improve students' language abilities through content-focused learning and teaching by purposefully ignoring the crust of content---language. It then presents the strange phenomenon of some universities or departments trying to use humanities-based instruction to achieve students' language goal. Subsequently the paper proposes its core opinion: It is infeasible to apply humanities-based instruction to achieve students' language goal for two reasons. One reason is that students can hardly be inspired to use related language as the chances of coming into contact with a certain humanistic subject in reality are slim. The other is that students have difficulty communicating in English about a certain humanist subject for its complicated ideas and specialized vocabulary. The infeasibility of applying humanities-based instruction to achieve students' language goal is also verified through the interview with several students of the writer. Despite the failure of humanitiesbased instruction to achieve students' language goal, some universities or departments favors humanities-based instruction either for teachers' personal purposes and interests or for the 
cultivation of the mind and the gain of ideas in the advanced stage of language study. There are two remedies for the misunderstandings and misuse of humanities-based instruction. One is to turn to theme-based instruction to improve students' language proficiency. The other is to specialize in a certain humanistic field for future career preparation or for pure interest. I hope this paper can shed light on the essence of humanities-based instruction.

\section{References}

[1]Brinton, D., Snow M. \& Wesche, M. Content-Based Language Instruction. New York: Newburry House, 1989.

[2]Krueger, M. \& Ryan, F. (eds). Language and Content Discipline and Content-Based Approaches to Language Study. Lexington, MA: DC Heath, 1993.

[3]Met, M. Learning Language through Content Learning Content through Language. Foreign Language Annals, 1991, (24/4).

[4]Chen Dongchun. College English Teaching and Teachers' Professional Development under CBI Theory. Technology Enhanced Foreign Language Education, 2014(3): 69.

[5]Wang Shixian, CBI_-The Trend of Reading Classes for English Majors. Foreign Language World, 1994(2): 28, 30. 